\title{
Neuroradiology Fellowship Case Requirements Need Reform
}

$\mathbf{P}$ ediatric radiology and neuroradiology have long been radiology subspecialties certified by the American Board of Radiology (ABR), and both pathways have been Accreditation Council for Graduate Medical Education (ACGME)-accredited for many years. The discipline of pediatric neuroradiology, however, remains unaccredited by the ACGME despite increased interest in postgraduate year 7-level pediatric radiology fellowships in recent years. Pediatric neuroradiologists who complete a pediatric neuroradiology fellowship must thus practice as neuroradiology faculty at an accredited program for at least 2 years before the subspecialty examination can be taken. Pediatric neuroradiologists in private practice unaffiliated with accredited training environments are ineligible for the neuroradiology subspecialty certificate even though they have completed a year of dedicated training.

Neuroradiologists were not long ago entrenched in interventional procedures, but noninvasive angiography using CT and MR imaging has greatly supplanted catheter-based cerebral angiography. The rise of endovascular surgical neuroradiology as a distinct ACGME-accredited fellowship-which can be undertaken by neurologists and neurosurgeons - has further reduced the volume of direct cerebral angiography performed by radiologists. Despite this reduction, ACGME requirements still mandate participation in 50 catheter-based angiographic procedures and 50 image-guided invasive procedures. ${ }^{1}$ Programs with as many as 8 fellows must thus have 400 catheter-based angiographic procedures available. Many neuroradiology programs now struggle to meet this requirement, and others report that participation is very loosely defined.

Case log requirements also include interpretation of 1500 neuroradiologic CT scans and 1500 neuroradiologic MR images. Review of a recent pediatric neuroradiology graduate's case log indicated $1321 \mathrm{CT}$ scans and $2920 \mathrm{MR}$ images interpreted. Exposure to CT examinations in the pediatric population is challenging, given the need to limit radiation, but pediatric CT examinations are often greater in complexity due to variable maturation of pediatric osseous structures and/or complicated craniofacial/temporal bone anomalies. ACGME neuroradiology case log require-

http://dx.doi.org/10.3174/ajnr.A5553 ments also include exposure to plain radiography, transcranial Doppler sonography, and fetal sonography. Pediatric neuroradiologists are more likely to be involved in these types of examinations than adult neuroradiologists.

The ABR requires that a fellowship or employment take place at an ACGME-accredited institution before the experience can be applied to a training requirement for a subspecialty examination. Of the 14 domestic pediatric neuroradiology fellowship programs advertised by the American Society of Pediatric Neuroradiology, ${ }^{2}$ all take place at facilities that train ACGMEaccredited fellows.

I posit that the ACGME should consider altering its case log requirements to allow greater flexibility. A reasonable solution would be to have 3 available pathways, including a general neuroradiology curriculum that includes the current number of diagnostic neuroradiology examinations but fewer required procedures; a neurointerventional radiology pathway that is more focused on procedural neuroradiology but may have slightly fewer diagnostic requirements; and a pediatric neuroradiology option that requires more MR imaging examinations but fewer CTs.

A procedure-focused neuroradiology pathway would allow neuroradiology programs to concentrate these cases among individuals likely to perform these procedures in practice while giving them an excellent foundation should they opt to pursue advanced neurointerventional training. It would allow those less interested in procedures to focus more on advanced diagnostic neuroradiology skills (eg, functional MR imaging, diffusion tensor imaging, perfusion imaging, and so forth) that are becoming more relevant. A pediatric-focused neuroradiology pathway would provide pediatric neuroradiologists an avenue to acquire recognition of their training while broadening employment opportunities for pediatric radiologists who have this extra year of training.

The time has come to reassess the objectives and goals of neuroradiology fellowship training with respect to the state of the art in modern neuroradiology practice. By allowing variations to case $\log$ requirements, we can train future neuroradiologists to adapt their training to fit their own interests as well as the needs of modern employers.

AJNR Am J Neuroradiol 39:E49-E50 Apr 2018 www.ajnr.org 


\section{REFERENCES}

1. Accreditation Council for Graduate Medical Education. ACGME Program Requirements for Graduate Medical Education in Neuroradiology. https://www.acgme.org/Portals/0/PFAssets/ProgramRequirements/ 423_neuroradiology_2017-07-01.pdf. Accessed November 1, 2017

2. American Society of Pediatric Neuroradiology. Pediatric Neuroradiol- ogy Fellowships and Training. http://aspnr.org/pediatric-neuroradiologyfellowships/. Accessed November 1, 2017

(1)C.M. Pfeifer

Department of Radiology University of Texas Southwestern Medical Center Dallas, Texas 